\title{
A!
}

This is an electronic reprint of the original article.

This reprint may differ from the original in pagination and typographic detail.

Rajamäki, Robin; Koivunen, Visa

\section{Sparse linear nested array for active sensing}

Published in:

25th European Signal Processing Conference (EUSIPCO 2017)

DOI:

10.23919/EUSIPCO.2017.8081555

Published: 01/01/2017

Document Version

Peer reviewed version

Please cite the original version:

Rajamäki, R., \& Koivunen, V. (2017). Sparse linear nested array for active sensing. In 25th European Signal Processing Conference (EUSIPCO 2017) (pp. 1976-1980). IEEE.

https://doi.org/10.23919/EUSIPCO.2017.8081555

This material is protected by copyright and other intellectual property rights, and duplication or sale of all or part of any of the repository collections is not permitted, except that material may be duplicated by you for your research use or educational purposes in electronic or print form. You must obtain permission for any other use. Electronic or print copies may not be offered, whether for sale or otherwise to anyone who is not an authorised user. 


\title{
Sparse Linear Nested Array for Active Sensing
}

\author{
Robin Rajamäki and Visa Koivunen \\ School of Electrical Engineering \\ Aalto University, 02150 Espoo, Finland \\ Email: robin.rajamaki@aalto.fi, visa.koivunen@aalto.fi
}

\begin{abstract}
Sparse sensor arrays can match the performance of fully populated arrays using substantially fewer elements. However, finding the array configuration with the smallest number of elements is generally a computationally difficult problem. Consequently, simple to generate array configurations that may be suboptimal are of high practical interest. This paper presents a novel closed-form sparse linear array configuration designed for active sensing, called the Concatenated Nested Array (CNA). The key parameters of the CNA are derived. The CNA is also compared to the optimal Minimum-Redundancy Array (MRA) in numerical simulations. The CNA is shown to require only about $10 \%$ more elements than the MRA in the limit of large apertures.
\end{abstract}

\section{INTRODUCTION}

Phased sensor arrays are a critical technology in many application areas including radar, wireless communication, medical imaging, radio astronomy, sonar and seismology [1]. Key advantages of sensor arrays include spatial selectivity and the capability to mitigate interference and improve signal quality. A major factor determining the cost of these arrays is the number of sensors, which may grow impractically high for large apertures with uniformly spaced elements. Sparse or thinned sensor arrays offer an attractive solution to this problem. A sparse array can achieve the same degrees of freedom (DOF) as a filled array with $N$ elements, using only $\mathcal{O}(\sqrt{N})$ elements [2]. The DOF determine e.g. the achievable point spread function in array imaging [3], the number of resolvable sources in direction-of-arrival (DOA) estimation [4], [5], or the beamforming capabilities of the array [6]. Mutual coupling may also be reduced due to the larger interelement distances in sparse arrays [7], [8].

Finding optimal sparse array geometries, such as the Minimum-Redundancy Array (MRA) [9], is generally a computationally hard problem, with no known polynomial time solution. This has led to the development of several suboptimal, but mathematically tractable array configurations [5], [8], [10]-[12]. However, most of these geometries have been developed for passive sensing (receive-only operation) and are therefore not directly suited for active sensing applications. Nonetheless, some exceptions exist. For example, optimal sparse linear arrays with spatially separated transmitting and receiving elements may be generated by simple interpolation [13]. Furthermore, it has been shown that placing elements on the convex boundary of an active planar array is effectively equivalent to filling the interior of the array with virtual elements [12], [14]. Although planar arrays are of considerable practical interest due to their capability of beamforming in both the azimuthal and elevational directions, linear arrays also have value in many applications, as well as in developing array processing methods and theory. In the past, some effort has been put in finding sparse linear geometries with colocated transceiver elements. In [15], the authors suggested extending the mid-sections of MRAs with a certain structure in order to create sparse arrays of increasingly larger aperture. Unfortunately, the number of elements $N$ in these Reduced Redundancy Arrays (RRAs) scales linearly with aperture $L$, although generally $N \propto \sqrt{L}$ should be achievable. A related case was studied in [16], [17], where MIMO arrays using second-order statistics were considered. However, the resulting optimization problem involved the difference sum co-array, whereas linear processing and the sum co-array [2], [15] are of main interest in this paper.

To address the aforementioned problems, this paper proposes a novel linear sparse array configuration called the Concatenated Nested Array (CNA) based on the Nested Array in [5]. A low-complexity method is introduced for generating the CNA for a desired aperture or number of elements. The performance of the CNA is analyzed both in closed-form and through numerical simulations. These analyses compare the geometrical efficiency of the CNA with the MRA through quantities such as redundancy and sparseness. The sum coarray of the CNA is also proven to be gapless.

The paper is organized into four sections. Section II provides a brief overview of the array processing model and the necessary definitions and concepts. Section III introduces the CNA and establishes its basic properties, such as aperture and number of elements. Section IV numerically compares the redundancy and sparseness of the CNA and MRA. Finally, section $\mathrm{V}$ summarizes the central findings of the paper.

\section{SIGNAL MODEL AND DEFINITIONS}

\section{A. Sparse arrays and the co-array}

In this paper, a sparse array is defined as a thinned Uniform Linear Array (ULA), i.e. an array whose elements are located on a regular grid with unit distance $d$, the inter-element spacing of the ULA. The task of sparse array design is to find a geometry that uses fewer elements than the ULA, without experiencing significant performance degradation.

The co-array is a virtual structure that arises in certain array processing tasks, such as imaging or DOA estimation [2]. Essentially, the co-array quantifies the spatial redundancy of the used array geometry at a given wavelength of operation. Under narrow-band, far-field assumptions, the grid of the physical array is usually normalized to yield a set of integers for sensor locations. As a result, the co-array of a linear array may be represented as set of integer value pairs, which denote the position and multiplicity of virtual array elements. When the co-array contains every integer between its endpoints, it is 
called contiguous. A sparse array with a contiguous co-array has the same number of DOF as the ULA of the same physical aperture. The arrays considered in this paper are restricted to those with contiguous sum co-arrays.

1) Sum co-array: The sum co-array appears in active array processing applications, such as linear array imaging [2]. In these cases, the support of the virtual array is given by the pairwise sums of element positions $\mathbf{d}_{i}, i=1,2, \ldots, N$. The support of the sum co-array is defined

$$
\mathcal{C}_{\Sigma}=\left\{\mathbf{d}_{n}+\mathbf{d}_{\tilde{n}}\right\} ; n, \tilde{n}=1,2, \ldots, N .
$$

For a linear array of aperture $L$, Eq. (1) reduces to a set of integers between 0 and $2 L$.

2) Difference co-array: The difference co-array arises in applications employing second-order statistics, such as DOA estimation [2]. The difference co-array is therefore primarily of interest in passive array processing. However, since the difference co-array conveniently captures the multiplicity of different inter-element spacings present in the array, it can be useful e.g. in assessing how much the array configuration is subject to mutual coupling, a major source of non-idealities in practical arrays. Similarly to Eq. (1), the support of the difference co-array is defined: $\mathcal{C}_{\Delta}=\left\{\mathbf{d}_{n}-\mathbf{d}_{\tilde{n}}\right\} ; n, \tilde{n}=1,2 \ldots N$. The difference co-array of a linear array spans the integers between $-L$ and $L$.

\section{B. Postage stamp problem}

The search of sparse linear arrays for active sensing is a specific case of the postage stamp problem from number theory. For a comprehensive review of the subject see [18]. The formulation of the postage stamp problem is briefly stated as follows: Given $k$ different stamps, the largest postage fare is sought so that each smaller fare can be represented as the sum of $h$ or less stamps. The fare and stamps are assumed to have integer values and the problem is often augmented to include a stamp of value 0 as well. An additive $h$-basis is a candidate solution to the postage stamp problem. Formally, an $h$-basis is a set of $k$ integers $A_{k}=\left\{0=a_{0}<a_{1}<\cdots<a_{k}\right\}$, such that $0,1, \ldots, n$ can be represented as the sum of at most $h$ (not necessarily distinct) elements of $A_{k}$. A solution is called restricted if $a_{k}=n / 2$, where $n=n(h, k)$ denotes the largest possible postage fare. Additionally, if a solution is extremal, then it achieves the largest possible $n$ for a fixed $k$. Finding extremal restricted additive 2-bases is equivalent to finding active linear MRAs. It should also be mentioned that finding restricted difference bases [19] is the passive sensing counterpart of finding restricted additive bases. The computational efforts for finding extremal 2-bases are primarily based on branch and bound tree searches with various pruning strategies [20]-[22]. The largest known restricted extremal 2-base produced by this type of search is $n(2,47)=734$ [23].

\section{Figures of merit of sparse arrays}

1) Redundancy: The ratio of the theoretical maximum and actual number of DOF of an array is quantified by redundancy. The sum co-array of an active array with $N$ co-located transmitters (Tx) and receivers $(\mathrm{Rx})$ cannot have more than $\sum_{i=0}^{N-1}(N-i)=\frac{1}{2} N(N+1)$ unique elements, or DOFs. If the aperture of the physical array is $L$, the sum co-array has $2 L+1$ unique elements in case it is contiguous. The redundancy of an active linear array with co-located $\mathrm{Tx} / \mathrm{Rx}$ elements and a contiguous sum co-array may therefore be defined as [15]:

$$
R=\frac{\frac{1}{2} N(N+1)}{2 L+1}=\frac{N(N+1)}{4 L+2} .
$$

Ideally $R=1$, but for practical arrays $R>1$. Different array configurations may also be compared with each other in a aperture independent manner using the asympotic redundancy:

$$
R_{\infty}=\lim _{N \rightarrow \infty} R=\lim _{N \rightarrow \infty} \frac{1}{4} \frac{N^{2}}{L(N)}=\lim _{k \rightarrow \infty} \frac{1}{2} \frac{k^{2}}{n(2, k)},
$$

where the ratio $k^{2} / n$ is commonly seen in the literature on additive bases [18]. The relationship between $k, n$ and the array parameters is $N=k+1$ and $L=n / 2$.

2) Element redundancy: The asymptotic ratio,

$$
\eta_{\infty}=\lim _{L \rightarrow \infty} \frac{N(L)}{N_{\mathrm{MRA}}(L)}=\sqrt{\frac{R_{\infty}}{R_{\infty, \mathrm{MRA}}}}
$$

quantifies the fraction of elements required by a given physical array configuration with respect to the minimum possible (i.e. the MRA) in the limit of large apertures. Although the element redundancy $\eta_{\infty}$ contains the same information as the asymptotic redundancy $R_{\infty}$, the interpretation of the former is more immediate. The element redundancy is given as an interval, since the asymptotic redundancy of the MRA is only known to lie within a certain range (see section II-D2).

3) Sparseness: Eq. (2) does not take into account the distribution of redundancies, which may be of interest when considering, for example, the mutual coupling between elements. From this point of view, the larger the displacements among the elements are, the less mutual coupling is expected. It is therefore reasonable to quantify the sparseness (or denseness) of the array. The number of unit spacings in the array gives a simple first-order approximation of denseness. Denoting the multiplicity of element $i$ in the difference co-array of a given physical array by $v_{\Delta}(i)$, the number of unit spacings is given by $v_{\Delta}(1)=v_{\Delta}(-1)$. In general, a configuration $a$ is considered sparser than $b$, if $v_{\Delta, a}(j)<v_{\Delta, b}(j)$ and $v_{\Delta, a}(i)=v_{\Delta, b}(i)$ for $i=0,1, \ldots, j-1$.

\section{Sparse array configurations}

1) Perfect array: The perfect array (PA) is an ideal sparse array that has a contiguous co-array and no redundancy. PAs only exist for $N \leq 2$ elements in the active case (and $N \leq 4$ in the passive case [9]), but they offer a closed-form expression for $L, N$ and $R_{\infty}$, whereas MRAs do not. These expressions are convenient for establishing bounds on the maximum aperture and minimum number of elements of realizable arrays. The aperture of the PA is simply given by the number of DOFs in the sum co-array: $L=N(N+1) / 2$. Consequently, the number of elements is $N=(\sqrt{8 L+1}-1) / 2$ and the redundancy, by definition $R=R_{\infty}=1$.

2) Minimum-Redundancy Array: Although generating MRAs is difficult, bounds on their asymptotic redundancy have been established. A lower bound on the largest integer $n$ that may be represented by a given extremal additive 2-base is $n \geq k^{2} 2 / 7+\mathcal{O}(k)$ [20]. Conversely, for $n$ sufficiently large it 


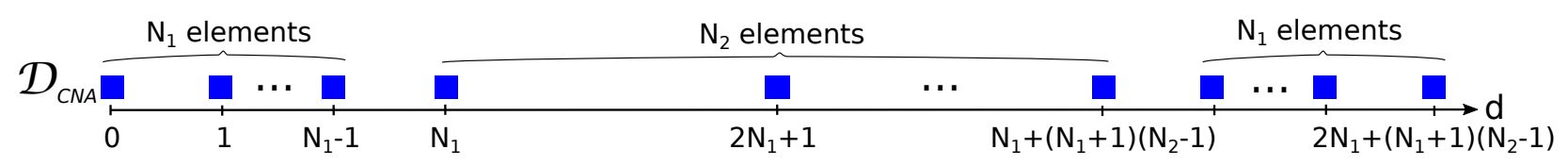

Fig. 1. Geometry of the Concatenated Nested Array (CNA). The CNA may be viewed as a Nested Array [5] and its mirror image placed in succession.

is shown in [24] that $n<k^{2}(1-0.1513) / 2$. Combining these two results with Eq. (3) bounds the asymptotic redundancy of the (active) MRA as follows:

$$
1.18 \leq R_{\infty} \leq 1.75
$$

MRAs may be generated for any number of elements $N \leq 48$ using the tables precomputed in [22], [23]. In case several configurations with the same redundancy and aperture are available, the sparsest one (according to the definition in section II-C3) is chosen.

3) Nested array: The Nested Array (NA) [5] is a low redundancy sparse linear array configuration for passive sensing, consisting of a dense and sparse ULA placed in succession. The inter-element spacings of the NA are given by $d \mathcal{D}_{\mathrm{NA}}=\left\{1^{\left(N_{1}\right)},\left(N_{1}+1\right)^{\left(N_{2}-1\right)}\right\}$, where the notation $x^{(y)}$ reads " $y$ repetitions of $x "$, and $N_{1}, N_{2} \in \mathbb{N}$ denote the number of elements in the two ULAs. The difference co-array of the NA has the convenient property of being contiguous.

\section{ConcatenAted Nested ARray}

\section{A. Array design}

The sum co-array of the Nested Array contains gaps, which could limit its applicability in active sensing application. In order to ensure sum co-array equivalence with the ULA, it is proposed here to place two NAs in a sequence, such that the second array is a mirror image of the first (Fig. 1). The mirroring ensures that the resulting array, referred to as the Concatenated Nested Array (CNA), has a contiguous sum co-array. This enables the CNA to e.g. achieve the same point spread function in imaging applications as the ULA of equivalent aperture.

Proposition: The sum co-array of the CNA is contiguous.

Proof: See Appendix.

Next the CNA is formally defined. Given $N_{1}, N_{2} \in \mathbb{N}$, the inter-element spacings of the CNA are given by

$$
d \mathcal{D}_{\mathrm{CNA}}=\left\{1^{\left(N_{1}\right)},\left(N_{1}+1\right)^{\left(N_{2}-1\right)}, 1^{\left(N_{1}\right)}\right\} .
$$

It follows from Eq. (6) that the aperture of the CNA is

$$
L=2 N_{1}+\left(N_{1}+1\right)\left(N_{2}-1\right) .
$$

Furthermore, the total number of elements is

$$
N=2 N_{1}+N_{2}
$$

Eq. (7) and (8) can be used to formulate an optimization problem for finding parameters $N_{1}, N_{2}$ that maximize the aperture $L$ for a given number of elements $N$ :

$$
\begin{aligned}
\underset{N_{1}, N_{2} \in \mathbb{N}}{\operatorname{maximize}} & N_{1} N_{2}+N_{1}+N_{2}-1 \\
\text { subject to } & 2 N_{1}+N_{2}=N .
\end{aligned}
$$

\section{B. Solution to relaxed problem}

Although (P1) is an integer program with no general closed-form solution, a bound on the maximum possible aperture may be found under the relaxation that $N_{1}, N_{2} \in \mathbb{R}_{+}$. Solving Eq. (8) for $N_{2}$ and inserting the result into Eq. (7) yields

$$
L=-2 N_{1}^{2}+(N-1) N_{1}+N-1,
$$

which is a concave function that is straightforward to maximize. The optimal parameter pair of the relaxed problem is

$$
\left\{\begin{array}{l}
N_{1}^{\star}=(N-1) / 4 \\
N_{2}^{\star}=(N+1) / 2
\end{array}\right.
$$

which yields the maximum aperture achievable by the CNA:

$$
L^{\star}=\left(N^{2}+6 N-7\right) / 8 .
$$

Equivalently, the minimum number of elements for a given aperture is

$$
N^{\star}=2 \sqrt{2} \sqrt{L+2}-3 .
$$

Using Eq. (11) and (3), the asymptotic redundancy of the CNA evaluates to $R_{\infty}=\lim _{N \rightarrow \infty} \frac{N^{2}}{4 N^{2} / 8}=2$. It also follows from Eq. (6), (10) and (12) that the number of unit spacings of the CNA is $v_{\Delta}(1)=2 N_{1}=(N-1) / 2=\sqrt{2} \sqrt{L+2}-2$.

\section{General solution}

Eq. (10) only produces integer valued solutions for every fourth odd $N$. As a result, the fractional solutions to the relaxed problem have to be projected to the set of integers. In order to find these remaining solutions, the closest integer to $N_{1}^{*}$ in Eq. (10) is selected, i.e.

$$
\left\{\begin{array}{l}
N_{1}=\lceil(N-1) / 4\rfloor \\
N_{2}=N-2 N_{1}
\end{array}\right.
$$

where $\lceil\cdot\rfloor$ denotes rounding to the nearest integer and $N_{2}$ is the solution to Eq. (8). Eq. (13) produces all feasible solutions to (P1). When a solution for a specific aperture $L$ is required, the CNA parameters $N_{1}$ and $N_{2}$ may be found by minimizing the number of elements for a target aperture instead:

$$
\begin{aligned}
\underset{N_{1}, N_{2} \in \mathbb{N}}{\operatorname{minimize}} & 2 N_{1}+N_{2} \\
\text { subject to } & 2 N_{1}+\left(N_{1}+1\right)\left(N_{2}-1\right)=L .
\end{aligned}
$$

Relaxing the integer constraint to $N_{1}, N_{2} \in \mathbb{R}_{+}$and solving (P2) for $N_{1}$ yields

$$
N_{1}^{*}=\frac{1}{\sqrt{2}} \sqrt{L+2}-1 .
$$

Given $L$, the following two step optimization procedure may be applied: Find the integer $N_{1} \in\{0,1, \ldots,\lfloor(L+1) / 2\rfloor\}$ $(\lfloor\cdot\rfloor$ is the floor operator) that i) has an integer-valued pair $N_{2}=\left(L-\left\lceil N_{1}\right\rfloor+1\right) /\left(\left\lceil N_{1}\right\rfloor+1\right) \in \mathbb{N}$ and ii) is closest to the ideal $N_{1}^{*}$ computed using Eq. (14). If no such 
parameter is found, then a solution for aperture $L$ does not exist. This procedure produces solutions of lower quality than Eq. (13), since it is possible to construct a CNA with larger aperture using the same number of elements. Consequently, only solutions to Eq. (13) are considered in the following.

\section{NUMERICAL RESULTS}

In this section, the redundancy and sparseness of the CNA and MRA are compared for $L \leq 400$. MRAs up to $L=367$ are taken from the tables in [22], [23]. A design with a low redundancy and as few unit spacings as possible is desired, since it requires fewer elements to construct and is expected to be less susceptible to mutual coupling.

Fig. 2 shows the CNA and MRA configurations for $L=58$, which is the smallest common value among the considered apertures for which the two solutions differ. In this case the MRA has $N=18$ and the CNA $N=19$ elements. The MRA is typically symmetric [22], [23], with a sparse mid-section and more densely spaced elements in both ends. The CNA also follows a similar pattern. In fact, the CNA is the MRA for all overlapping solutions when $L \leq 58$. The symmetrical physical arrays in Fig. 2 give rise to the symmetrical contiguous sum co-arrays shown in Fig. 3.

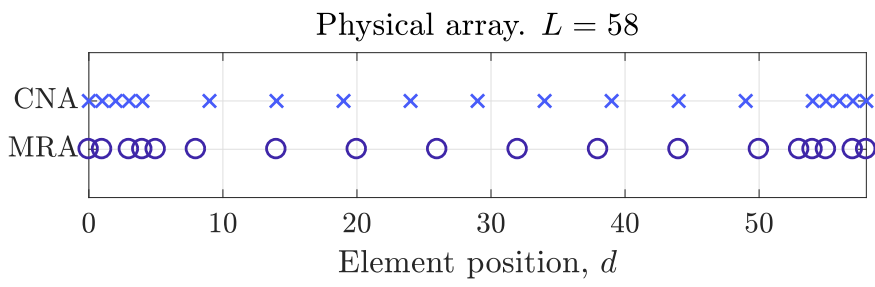

Fig. 2. Array configurations for aperture $L=58$. The MRA has 18 and the CNA 19 elements. In general, the MRA is symmetrical with a sparse mid section. The CNA has a structure very similar to the MRA.

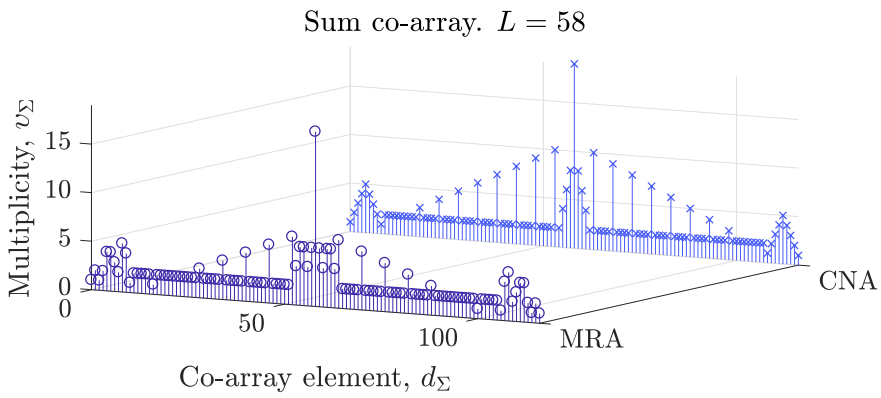

Fig. 3. Sum co-array of the array configurations in Fig. 2. Since the co-arrays do not contain holes, the MRA and CNA have the same sum co-array support as the ULA of equivalent physical aperture ( $L=58$ in this case).

Fig. 4 shows the redundancies of the generated CNAs and MRAs. The CNA exceeds $R=1.8$ at $L \approx 250$ and slowly converges towards its asymptotic value of 2 . The MRA achieves $R=1.6$ for approximately the same aperture and does not increase for the largest known instances, which is a surprising property. Clearly, the lower bound on the asymptotic redundancy $R_{\infty}>1.18$ given in Eq. (5) is quite loose and may be empirically tightened to roughly $R_{\infty} \geq 1.6$.

Fig. 5 shows the number of unit spacings $v_{\Delta}(1)$ in the two arrays. In case of the CNA, $v_{\Delta}(1) \propto \sqrt{L}$ as shown in

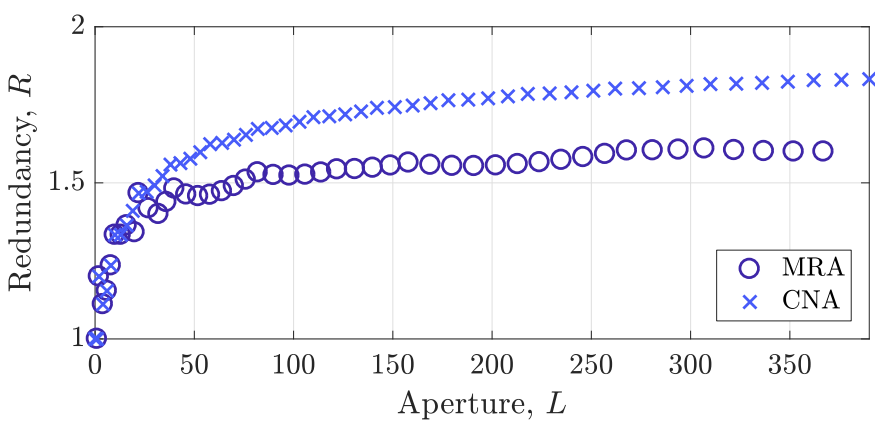

Fig. 4. Redundancies of the generated configurations. The redundancy of the CNA converges towards its asymptotic value of 2 , whereas for the MRA this value lies approximately between 1.6 and 1.75 .

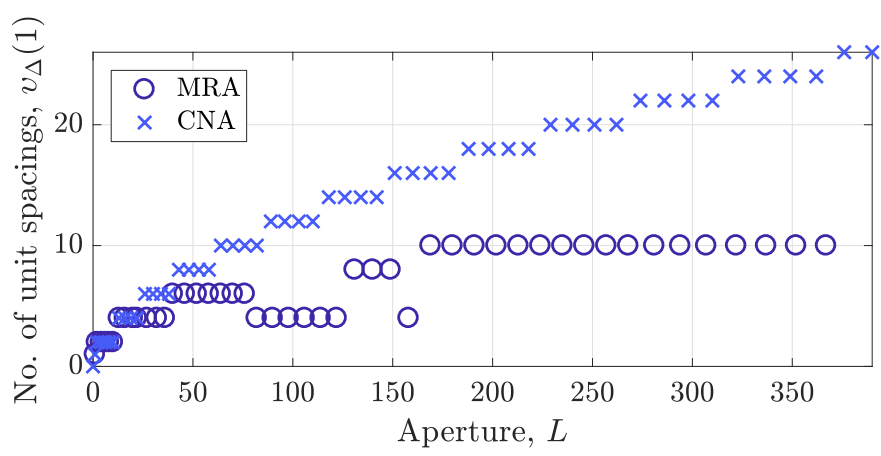

Fig. 5. Number of unit spacings in the MRA and CNA. This quantity increases $\propto \sqrt{L}$ for the CNA, but changes erratically for the MRA.

section III-B. Although the MRA has a lower number of unit spacings for all inspected configurations, this quantity seems unpredictable. In particular, $v_{\Delta}(1)$ does not monotonically increase as a function of $L$, but sometimes abruptly decreases and actually remains at $v_{\Delta}(1)=10$ between $L=169$ ( $N=32$ ) and $L=367(N=48)$. It would be interesting to study how the quantity changes for MRAs of larger aperture.

A summary of the main properties of the CNA is given in Table I. The parameters of the PA are listed as a reference, due to the lack of closed-form solutions for the MRA. Table I also shows the asymptotic element redundancy of the CNA, which is roughly 1.1 times that of the MRA. For comparison, the corresponding figure for the RRA (see section I) is not even finite. In other words, the CNA requires asymptotically only $10 \%$, and the RRA infinitely, more elements than the MRA. Curiously, the difference between the MRA and PA is $\approx 22 \%$, although obviously the latter is not realizable in practice.

\section{CONCLUSION}

This paper presented a novel sparse linear array configuration for active sensing called the Concatenated Nested Array. Closed-form expressions for the aperture, number of elements and number of unit spacings in the array were introduced. The CNA is simple to generate for any aperture or number of elements. It also has a contiguous sum co-array, which means that it has the same degrees of freedom as the ULA of equivalent aperture. Consequently, the CNA may e.g. attain any point spread function that the ULA can in imaging applications. Since the CNA uses significantly fewer elements 
TABLE I. PROPERTIES OF THE CNA IN COMPARISON WITH THE PA AND MRA. THE CNA ONLY REQUIRES APPROXIMATELY 10\% MORE ELEMENTS THAN THE MRA. THE PA IS MOSTLY NON-REALIZABLE IN PRACTICE, BUT ITS PARAMETERS HAVE CONVENIENT CLOSED-FORM EXPRESSIONS.

\begin{tabular}{|c|c|c|c|c|c|}
\hline Array config. & Max. aperture, $L$ & Min. no. of elements, $N$ & No. of unit spacings, $v_{\Delta}(1)$ & Asymptotic redundancy, $R_{\infty}$ & Element redundancy, $\eta_{\infty}$ \\
\hline Perfect Array (PA) & $\left(N^{2}+N\right) / 2$ & $(\sqrt{8 L+1}-1) / 2$ & 1 & 1 & $0.76-0.79$ \\
\hline Minimum-Redundancy Array (MRA) & N/A & N/A & N/A & $1.6-1.75$ & 1 \\
\hline Concatenated Nested Array (CNA) & $\left(N^{2}+6 N-7\right) / 8$ & $2 \sqrt{2} \sqrt{L+2}-3$ & $\sqrt{2} \sqrt{L+2}-2$ & 2 & $1.07-1.12$ \\
\hline
\end{tabular}

than the ULA, considerable cost reductions may be achieved. In fact when the aperture grows large, the CNA requires only approximately $10 \%$ more elements than the MinimumRedundancy Array.

\section{APPENDIX}

\section{PROOF OF CONTIGUOUS SUM CO-ARRAY OF CNA}

The sum co-array can be decomposed into the union of self and cross co-arrays of two sub-arrays, which are acquired by dividing the CNA at its geometrical midpoint into two nonoverlapping parts. It is shown next that the sum co-array of the CNA is contiguous, since the union of the component coarrays is contiguous: Let the element positions of the sparse array be encoded into a binary sequence $b[i], i=0,1, \ldots, L$, where $b[i]=1$ if position $i$ contains an element and $b[i]=0$ if the position is empty. Sequence $b$ may also be written as the sum of the two sub-array sequences $b_{1}$ and $b_{2}$, i.e. $b[i]=b_{1}[i]+b_{2}[i]$. Since the sum co-array, $c$, is the convolution of transmitting and receiving elements [2], it follows that $c=b * b=\left(b_{1}+b_{2}\right) *\left(b_{1}+b_{2}\right)=b_{1} * b_{1}+2 b_{1} * b_{2}+b_{2} * b_{2}$, where ' $*$ ' denotes convolution. In other words, the sum coarray is the sum of the self co-arrays $c_{1}=b_{1} * b_{1}$ and $c_{2}=b_{2} * b_{2}$, and the cross co-array $c_{12}=2 b_{1} * b_{2}$. Let $\mathcal{C}_{k}=\left\{i \mid c_{k}[i] \neq 0\right\}$ denote the support of co-array $c_{k}$, i.e. the positions in which it has non-zero values. Furthermore, let $\mathcal{C}_{k}$ be the contiguous support, i.e. the largest sequence of successive integers in $\mathcal{C}_{k}$. In the case of odd $N$, the CNA has an element at $L / 2$ and the contiguous parts of the three component co-arrays may be deduced from the geometry to be $\tilde{\mathcal{C}}_{1}=\left\{0, \ldots, L / 2+N_{1}\right\}, \tilde{\mathcal{C}}_{12}=\left\{L / 2+N_{1}+1, \ldots, 3 / 2 L\right\}$ and $\tilde{\mathcal{C}}_{2}=\{3 / 2 L+1, \ldots, 2 L\}$. Since $\tilde{\mathcal{C}}=\tilde{\mathcal{C}}_{1} \cup \tilde{\mathcal{C}}_{12} \cup \tilde{\mathcal{C}}_{2}=$ $\{0,1 \ldots, 2 L\}=\mathcal{C}$, the sum co-array is contiguous for odd $N$. Similarly, when $N$ is even the component sub-arrays have the contiguous ranges $\tilde{\mathcal{C}}_{1}=\left\{0, \ldots, L / 2+\left(N_{1}+1\right) / 2\right\}$, $\tilde{\mathcal{C}}_{12}=\left\{L / 2+\left(N_{1}+1\right) / 2, \ldots, 3 / 2 L+\left(N_{1}+1\right) / 2\right\}$ and $\tilde{\mathcal{C}}_{2}=\left\{3 / 2 L+\left(N_{1}+1\right) / 2, \ldots, 2 L\right\}$. Therefore the co-array is contiguous also for even $N$.

\section{REFERENCES}

[1] H. L. Van Trees, Optimum Array Processing, Part IV of Detection, Estimation, and Modulation Theory. Wiley-Interscience, 2002.

[2] R. T. Hoctor and S. A. Kassam, "The unifying role of the coarray in aperture synthesis for coherent and incoherent imaging," Proceedings of the IEEE, vol. 78, no. 4, pp. 735-752, Apr 1990.

[3] F. Ahmad, G. J. Frazer, S. A. Kassam, and M. G. Amin, "Design and implementation of near-field, wideband synthetic aperture beamformers," IEEE Transactions on Aerospace and Electronic Systems, vol. 40, no. 1, pp. 206-220, Jan 2004

[4] R. T. Hoctor and S. A. Kassam, "High resolution coherent source location using transmit/receive arrays," IEEE Transactions on Image Processing, vol. 1, no. 1, pp. 88-100, Jan 1992.

[5] P. Pal and P. P. Vaidyanathan, "Nested arrays: A novel approach to array processing with enhanced degrees of freedom," IEEE Transactions on Signal Processing, vol. 58, no. 8, pp. 4167-4181, Aug 2010.
[6] K. Adhikari, J. R. Buck, and K. E. Wage, "Extending coprime sensor arrays to achieve the peak side lobe height of a full uniform linear array," EURASIP Journal on Advances in Signal Processing, vol. 2014, no. 1, pp. 1-17, 2014.

[7] E. BouDaher, F. Ahmad, M. G. Amin, and A. Hoorfar, "Mutual coupling effect and compensation in non-uniform arrays for direction-of-arrival estimation," Digital Signal Processing, pp. -, 2016.

[8] C. L. Liu and P. P. Vaidyanathan, "Super nested arrays: Linear sparse arrays with reduced mutual coupling - part i: Fundamentals," IEEE Transactions on Signal Processing, vol. 64, no. 15, pp. 3997-4012, Aug 2016.

[9] A. Moffet, "Minimum-redundancy linear arrays," IEEE Transactions on Antennas and Propagation, vol. 16, no. 2, pp. 172-175, Mar 1968.

[10] D. A. Linebarger, I. H. Sudborough, and I. G. Tollis, "Difference bases and sparse sensor arrays," IEEE Transactions on Information Theory, vol. 39, no. 2, pp. 716-721, Mar 1993.

[11] P. P. Vaidyanathan and P. Pal, "Sparse sensing with co-prime samplers and arrays," IEEE Transactions on Signal Processing, vol. 59, no. 2, pp. 573-586, Feb 2011.

[12] R. J. Kozick and S. A. Kassam, "Linear imaging with sensor arrays on convex polygonal boundaries," IEEE Transactions on Systems, Man, and Cybernetics, vol. 21, no. 5, pp. 1155-1166, Sep 1991.

[13] G. R. Lockwood, P.-C. Li, M. O’Donnell, and F. S. Foster, "Optimizing the radiation pattern of sparse periodic linear arrays," IEEE Transactions on Ultrasonics, Ferroelectrics, and Frequency Control, vol. 43, no. 1, pp. 7-14, Jan 1996.

[14] R. J. Kozick and S. A. Kassam, "Synthetic aperture pulse-echo imaging with rectangular boundary arrays," IEEE Transactions on Image Processing, vol. 2, no. 1, pp. 68-79, Jan 1993.

[15] R. T. Hoctor and S. A. Kassam, "Array redundancy for active line arrays," IEEE Transactions on Image Processing, vol. 5, no. 7, pp. 1179-1183, Jul 1996.

[16] C.-Y. Chen and P. P. Vaidyanathan, "Minimum redundancy MIMO radars," in IEEE International Symposium on Circuits and Systems, ISCAS 2008, May 2008, pp. 45-48.

[17] C. C. Weng and P. P. Vaidyanathan, "Nonuniform sparse array design for active sensing," in Conference Record of the Forty Fifth Asilomar Conference on Signals, Systems and Computer, ASILOMAR 2011, Nov 2011, pp. 1062-1066.

[18] R. Alter and J. A. Barnett, "A postage stamp problem," The American Mathematical Monthly, vol. 87, no. 3, pp. 206-210, 1980.

[19] J. Leech, "On the representation of 1, 2, , n by differences," Journal of the London Mathematical Society, vol. s1-31, no. 2, pp. 160-169, 1956.

[20] S. Mossige, "Algorithms for computing the h-range of the postage stamp problem," Mathematics of Computation, vol. 36, no. 154, pp. 575-582, 1981.

[21] M. F. Challis, "Two new techniques for computing extremal h-bases ak," The Computer Journal, vol. 36, no. 2, pp. 117-126, 1993.

[22] J. Kohonen, "A meet-in-the-middle algorithm for finding extremal restricted additive 2-bases," Journal of Integer Sequences, vol. 17, no. 2, p. 3,2014

[23] — , "Early pruning in the restricted postage stamp problem," arXiv preprint arXiv:1503.03416, 2015.

[24] L. Moser, J. R. Pounder, and J. Riddell, "On the cardinality of h-bases for n," Journal of the London Mathematical Society, vol. 1, no. 1, pp. 397-407, 1969. 